\title{
Stressful life events and maltreatment in conversion (functional neurological) disorder: systematic review and meta-analysis of case- control studies
}

Lea Ludwig, Joëlle A. Pasman, Timothy Nicholson, Selma Aybek, Anthony S. David, Sharon Tuck, Richard A. Kanaan, Karin Roelofs, Alan Carson, Jon Stone

Lea Ludwig, Department of Clinical Neurosciences, Western General Hospital, Edinburgh, EH4 2XU, UK

Department of Clinical Psychology and Psychotherapy, Universität Hamburg, Germany

Joëlle A. Pasman, Behavioural Science Institute, Developmental Psychopathology, Radboud University

Timothy Nicholson, Section of Cognitive Neuropsychiatry, Institute of Psychiatry Psychology \& Neuroscience, King's College,

London, UK

Selma Aybek, Section of Cognitive Neuropsychiatry, Institute of Psychiatry Psychology \& Neuroscience, King’s College, London, UK

Laboratory for Behavioral Neurology and Imaging of Cognition, Fundamental Neurosciences Department, Geneva University,

Geneva, Switzerland

Anthony S. David, Section of Cognitive Neuropsychiatry, Institute of Psychiatry Psychology \& Neuroscience, King's College, London, UK

Sharon Tuck, Epidemiology and Statistics Core, Edinburgh Clinical Research Facility, UK

Richard A. Kanaan, Department of Psychiatry, University of Melbourne, Austin Health, Heidelberg, Victoria, Australia, Florey Institute for Neuroscience and Mental Health, Heidelberg, Victoria, Australia

Karin Roelofs, Donders Institute for Brain Cognition and Behaviour \& Behavioural Science Institute, Radboud University Nijmegen Alan Carson, Department of Rehabiltation Medicine and Department of Clinical Neurosciences, NHS Lothian, Centre for Clinical Brain sciences, University of Edinburgh, UK

Jon Stone, Department of Clinical Neurosciences, Centre for Clinical Brain sciences, University of Edinburgh, UK T: +44 (0)131 5371167

Corresponding author: Lea Ludwig, lea.ludwig@posteo.de

Keywords: conversion disorder, functional neurological symptom disorder, psychogenic, life events, stress, trauma, maltreatment, abuse, neglect, systematic review, meta-analysis, aetiology, non-epileptic seizures, hysteria, dissociative, functional movement disorder

Word Count excluding abstract and tables: 4.276 


\section{Abstract}

\section{Background}

Stressful life events and maltreatment have traditionally been considered critical in the development of conversion (functional neurological) disorder (FND), but the evidence underpinning this association is less clear. We aimed to systematically assess the association between stressors and FND.

\section{Methods}

We systematically reviewed controlled studies reporting stressors occurring in childhood or adulthood, such as stressful life events and maltreatment (including sexual, physical abuse and emotional neglect) and FND. We conducted a meta-analysis, with assessments of methodology, sources of bias and sensitivity analyses.

\section{Findings}

Thirty-four case-control studies were eligible, including 1405 patients. Studies were of moderate to low quality. The frequency of childhood and adulthood stressors was increased in cases compared to controls. Odds ratios were higher for emotional neglect in childhood $449 \%$ vs $20 \%$ - OR $5 \cdot 6[2 \cdot 4-13 \cdot 195 \% \mathrm{CI}])$ vs sexual (30\% vs $12 \%$ - OR $3 \cdot 3[2 \cdot 2-4 \cdot 895 \% \mathrm{CI}]$ ) or physical abuse $(30 \%$ vs $12 \%$ - OR $3 \cdot 9[2 \cdot 2-7 \cdot 2$ 95\% CI]. An association with stressful life events preceding onset (OR 2·8[1.4-6.0 95\% CI]) was stronger in studies with better methodology (OR 4·3[1·4$13 \cdot 2$ 95\% CI]). There was significant heterogeneity between studies. Thirteen studies that specifically examined the question all found a proportion of FND patients reporting no stressor.

\section{Interpretation}

Stressful life events and maltreatment are significantly more common in FND than in controls. Emotional neglect carried a higher risk than traditionally emphasised sexual and physical abuse, but many cases report no stressors. This supports DSM- 5 changes to diagnostic criteria; 
stressors whilst aetiologically relevant are not a core diagnostic feature. This has implications for ICD-11.

\section{Funding}

None

\section{Introduction}

Conversion (functional neurological symptom) disorder (DSM-5 [FND]) refers to the experience of neurological symptoms in the absence of neurological disease encompassing symptoms such as limb weakness, seizures and movement disorders. Such disorders are one of the most common reasons for neurological referral (16\% of new referrals $)^{1}$ and as disabling and distressing as neurological counterparts such as multiple sclerosis or epilepsy². Traditionally, the disorder has been diagnosed on the absence of neurological disease and that "conflicts or other stressors precede the initiation or exacerbation of the symptom or deficit"3. However, the most recent edition of DSM-5 dropped the association with conflicts or other stressors as an explicit diagnostic criterion and emphasised the need to find positive clinical features such as Hoover's sign in functional leg weakness or a sudden prolonged motionless unresponsive episode with eyes closed in dissociative (non-epileptic) seizure. This change has not been universally welcomed and it is uncertain if ICD-11 will follow suit.

Stressors, either recent life events or maltreatment around the time of symptom onset or historical stressors, in particular childhood sexual abuse, have been considered key aetiological factors of FND since the time of Briquet's 1859 Treatise on hysteria4. In 1895 Breuer and Freud described the processes by which such psychological stress was converted into physical symptoms in their seminal Studies on Hysteria', giving the condition its name 'conversion disorder' and an aetiological theory that remains the bedrock of practice for the majority of clinicians to the current day.

There have however been critics of the conversion hypothesis who have commented that the empirical evidence to support the hypothesis is lacking and that the dominance of the theory distorts clinician's appreciation of the limitations of the available literature and inhibits the development of alternate or expanded models ${ }^{6}$.

Previous reviews summarising studies of stressors, including maltreatment and stressful life events, in FND have either not been systematic ${ }^{6}$, or have only reviewed non-epileptic seizures 
(NES) $)^{7,8}$ or childhood sexual abuse ${ }^{7}$. These reviews suggested an association of stressors and FND but were of limited scope. Looking at more broad phenotypes, reviews of somatic symptom disorders have notionally included FND, but either failed to identify much of the existing primary literature ${ }^{9}$ or were focused on functional somatic syndromes such as irritable bowel syndrome or chronic fatigue which overlap with but are different from FND ${ }^{10}$.

Technically, the study of maltreatment - here used as an umbrella term for sexual and physical abuse as well as emotional neglect - and stressful life events is challenging for many reasons. This includes patients' willingness to disclose sensitive information (and possibly even awareness of it or of its potential relevance), recall bias, difficulty determining over what time frame stressors are relevant, whether those that are present are aetiologically relevant, and the selection of appropriate controls. The use and selection of control groups is of particular importance as the rates of recent and historical stressors vary in different clinical (whether psychiatric or neurological) and healthy populations.

Furthermore, the descriptive terminology is at times ill-defined and in conducting a systematic review, one is in part dependent on the definitions used in individual studies. Thus, during the process of data amalgamation, it becomes inevitable that compromises are made between the uniqueness of an individual event and its psychological context, and the need to impose a taxonomy to allow quantitative study. We have developed a glossary of terminology that, whilst imperfect, allows for clarity and reproducibility (Appendix A).

We aimed to conduct a definitive systematic review of the association between childhood and adult stressful life events and maltreatment and conversion (functional neurological) disorder by reviewing all quantitative case-control studies since 1965 and comparing rates in FND populations with those in healthy, neurological or psychiatric disorder control populations. We excluded physical injury, physiological events or diseases as we have previously described their relationship to FND in prior systematic reviews and prospective studies $2,11,12$.

Whilst setting our aims we were cognisant of two further arguments. One is that one can only measure reported life events and maltreatment. Different techniques may result in better or poorer reporting, but ultimately there may be a distortion between what was reported and what occurred. Second, it has been argued that it is misleading to think about stressful life events and maltreatment and it is the patient's inner psychological state that matters, which some say can only be uncovered by prolonged psychotherapy. This is exemplified by one of Freud's original 
cases of treated hysteria, 'Fräulein Elisabeth von R'5, Freud considered the stressor was having romantic feelings for her brother in law, which the patient always denied. She also disputed Freud's assertion that she recovered from her FND symptoms. The truth of the matter is unresolved. Our view was a pragmatic one, that it would be very difficult to test subjective evaluation of emotions in a quantitative study and, more importantly, there were currently no empirical case control data of this type in FND that was suitable for quantitative meta-analytic evaluation. Our study therefore evaluated the occurrence of reported events.

\section{Methods}

\section{Search Strategy}

We searched the databases PubMed and Science Direct and the reference lists of eligible studies and reviews ${ }^{13,14}$ from 1965 to end 2016. Search terms were ("psychogenic" OR "conversion disorder" OR "non-epileptic") AND ("abuse" OR "life event") AND ("control” OR "controlled" OR "case-control").

\section{Study selection}

Studies were included if the following criteria were met: 1) they report on patients with conversion (functional neurological) disorders, described as functional, non-organic, psychogenic, hysterical or conversion disorder; 2) they report data comparing cases with at least one control group on the type, severity, frequency or temporal relationship of maltreatment or stressful life events, experienced in childhood or adulthood;3) the size of each group was at least 10 . We included studies in paediatric as well as adult populations. Where there were multiple publications from the same study, we chose the one with the more complete primary outcomes. Studies were excluded: 1) when the data of interest were presented only with p-values but with no numerical values in each group; 2) when the same data had been reported previously; 3) when studies were not available in English. Figure 1 outlines a flow diagram of the systematic review.

Figure 1

\section{Data Extraction}

All primary studies were reviewed by one author (LL). A second author checked the data (JP) and any discrepancies were arbitrated by two others (AC and JS). We collected data regarding 1) the setting of the samples; 2 ) the nature of case and control groups; 3 ) the sex and age of 
patients and controls; 4) the instruments used to measure stressors; 5) the data on stressors; and 6) the data from those studies that stated explicitly that the person has not experienced any maltreatment or stressful life event.

\section{Quality Assessment}

Methodological quality of eligible studies was assessed using an adaptation of the NewcastleOttawa Quality Assessment Scale for case-control studies ${ }^{15}$. The scale was adapted in keeping with Paras et al.9. Individual quality items are listed in Table 1. The quality was assessed twice (by LL and JP) and any disagreements were resolved by a further author (AC).

\section{Data analysis}

We calculated odds ratios (OR) with 95\% confidence intervals (CI) for both dichotomous and continuous data. We used statistical approaches described by Borenstein and colleagues that allow data pooling 16 . Furthermore, a proportion meta-analysis summary statistic for dichotomous data was used. Subgroup and sensitivity analyses were planned for the following grouping variables: study quality (median split of rating on quality scale, high vs low); type of control group (neurological vs psychiatric disorder vs healthy control); age of population studied (children vs adults); type of symptom (NES vs the rest); the time period where stressors took place; and setting of the study (patients recruited in neurology vs psychiatry settings). We ran fixed- and random-effect models using the software StatsDirect (Version 3.1.12) ${ }^{17}$. We quantified heterogeneity ${ }^{18}$ using a random effects model, and publication bias using the Egger bias statistic as well as inspection of funnel plots ${ }^{17}$. Where more than one set of data from an individual study could be included in a summary meta-analysis we used a hierarchy to choose which set be used in order to avoid duplication in the summary statistic as follows: stressful life events (data from more recent time points first), childhood stressful life events, sexual abuse, physical abuse, emotional neglect, neurological control group, psychiatric control group, healthy control group.

Finally, we calculated population attribution fractions around the main estimates ${ }^{19}$ taking data from a range of differing sources offering estimates based mainly on high quality systematic reviews and meta-analyses of population prevalence ${ }^{20-26}$.

\section{Results}




\section{Included studies}

In total 34 case-control studies met the inclusion criteria, providing stressful life events data for 1405 FND patients and 2227 controls which included healthy subjects as well as subjects with neurological disease and psychiatric disease (Table 2). In 24 studies data were presented on patients with NES $27-50$, five studies reported on general or mixed FND ${ }^{51-55}$, three studies provided data on Functional Motor Disorders (FMD) ${ }^{56-58}$, and two on Functional Voice Disorder (FVD - also known as functional dysphonia) ${ }^{59,60}$.

Thirty-one studies included adult subjects (mean age 37.1 yrs, range 18-77yrs) 27-42,44-46,48-52,5460, whereas the remaining three studies came from a paediatric setting (mean age $13.7 \mathrm{yrs}$, range 9-18 yrs $)^{43,47,53}$. In both the cases and control samples, most subjects were female $(79.7 \%$ cases vs $72.2 \%$ controls). In 25 out of 34 studies the patients were recruited from a neurology setting27,29-32,34,35,37-41,43-50,52,55,57,58,60, eight came from psychiatry28,33,36,42,51,53,54,56 and one study came from a mixed setting59. Eighteen studies out of 34 compared the functional patient group with a neurological disease control group27,29,30,32,34,35,37-40,44-46,48,50-52,58, mainly with epilepsy; seven studies with healthy controls $28,31,42,43,53,55,60$ and two studies with other psychiatric disorders control group ${ }^{36,54}$. In the remaining seven studies, data were presented deriving from a comparison with two control groups concurrently (most times including a healthy and a neurological control group) $33,41,47,49,56,57,59$.

Fourteen studies reported whether stressors had taken place at any moment in life ${ }^{30-}$ $33,35,36,38,39,41,48,51,56,59,60$. Two studies specifically reported on stressors in adulthood 49,52 , while eleven studies reported on those having occurred during childhood $27-29,37,40,42-44,47,53,58$. Seven studies presented separate rates for stressors occurred during childhood and for those occurred during lifetime or adulthood $34,45,46,50,54,55,57$. Nine studies specified the temporal relationship of life events with symptom onset $34,35,47,51,52,56,59-61$.

\section{Quality of Studies}

\section{Samples}

All of the studies assessed stressors retrospectively. Study setting was either neurology clinic, psychiatry clinic or other. Twenty studies recruited a consecutive sample $27,29,30,33-37,40,41,45-47,49,51-$ 53,58-60. In 27 studies the diagnosis was made by a specialist27,29-31,34-41,43-49,53-60. Fourteen adult sample studies reported symptom duration (excluding studies using symptom duration as an in- or exclusion criterion; mean=77.1 months, $S D=58 \cdot 2)^{28,31,34,38-42,47-50,54}$. Of those, eight studies 
compared symptom duration between cases and controls (three of them showing a significant difference ${ }^{39,49,50}$ ) but none tried to match controls on basis of symptom duration.

Assessment of reported stressors

In nine studies the interviewer (for outcome) was blind to the diagnosis $27,30,32,35,37,39,47,54,56$. The Life Events and Difficulties Schedule is often regarded as the 'gold standard' for such assessments in this field as it comprises of a detailed interview designed to detect a wide array of events but these are then rated blind and contextualized to subjects' life and circumstances to measure potential impact. Only three studies used the LEDS interview $56,59,60$. The majority of the 34 studies used standardized, structured questionnaires $27,31,34,35,37,38,42-46,49,50,55,57,58 \quad(n=15)$ or standardized interviews $29,39,41,48,51,53,61(n=7)$ to assess stressors. Four studies used owndesigned interviews ${ }^{30,32,36,40}$, one an own-designed questionnaire ${ }^{47}$ and two used case record data $^{33,51}$. Two studies did not report how stressors were assessed ${ }^{28,52}$.

The overall quality varied considerably among studies, ranging from 2-8 (with a possible maximum score of 11) on our modified Newcastle Ottawa scale (Table 1). The median score was 5 and there was variability in study quality $(\mathrm{IQR}=2)$.

Table 1

Table 2

\section{Meta-analysis of data}

The association of reported stressors and the occurrence of FND

Figure 2 and Table 3 present the meta-analysis of the data. Figure 2 shows the data (both continuous and dichotomous) in the form of odds ratios according to type of stressor and other study characteristics. Heterogeneity was high for nearly all analyses, so random effects analyses are presented throughout. Data for sensitivity analyses are presented together. Table 3 gives summary statistics for the dichotomous data where available. In summary, we found higher rates of reported stressors, both recent and remote, in patients with FND compared to controls. The risk was higher for childhood onset symptoms than in adult life. The odds ratio was higher for emotional neglect than for experiences of either physical or sexual abuse.

Figure 2

Table 3 
What proportion of FND cases did not report any form of stressor?

Calculating what proportion of cases of FND had not experienced stressors was less straightforward. The majority of studies only reported the rates of individual stressors found, but obviously if it is reported that, say, $34 \%$ of subjects were sexually abused one cannot impute that $66 \%$ suffered from no other form of stressor. Only 13 of the 34 studies presenting dichotomous data reported that they had systematically ascertained that the subjects had not experienced either severe life events, assessed by the LEDS, or any subtype of maltreatment 29,30,35,38,41,45,48,50,53,54,56,59,60 (Table 4, Figure 3). However, it was clear that the rigour underpinning the assessment of 'no stressor', or indeed what was meant by 'no stressor', was variable and we divided these 13 studies according to the methodology used. Three studies used the Life Events and Difficulties Scale (LEDS), one examined FND patients ${ }^{56}$ with $16 \%$ reporting no severe events, and two examined functional dysphonia patients ${ }^{59,60}$ finding conflicting results of $26 \%$ and $77 \%$ reporting no severe life events. Five studies $30,45,48,53,54$ examined a wide range of stressors but used a clinical interview rather than a structured inquiry about the experience of stressors (no stressful life events or maltreatment rates of 14\%, 15\%, 25\%, 51\%, $68 \%$ ). Two studies looked at all forms of maltreatment including sexual abuse, physical abuse and emotional neglect, but not stressful life events, finding no exposure rates of $56 \%{ }^{38}$ and $70 \%{ }^{41}$, and three studies $29,35,50$ offered data only on those who had not suffered physical and or sexual abuse with rates of $0 \%, 56 \%$ and $68 \%$.

Table 4

Figure 3

Assessment of specific risks at population level

Population attribution fraction (PAF) ${ }^{62}$ is a measure of the contribution of a risk factor to a disease or a death at a population rather than individual level. PAF is the proportional reduction in population disease or mortality that would occur if exposure to a risk factor were reduced to an alternative ideal exposure scenario (eg. no tobacco use). It gives a measure of the impact of a given aetiological exposure based on the frequency of its occurrence in the population as a whole and its effect in increasing the relative risk to an indvidual. We found that physical abuse had a greater aetiological impact on the development of FND with a PAF $17 \%$ of cases if it occurred in childhood and 15\% in adulthood, assuming a causal relationship, than sexual abuse, and to a lesser extent emotional neglect, as physical abuse is more prevalent in the population in general (Table 3). 


\section{Sensitivity Analysis}

We hypothesised that various methodological issues related to the nature of the symptom, population recruited, choice of control group, the assessed time period and the quality of the studies, could affect the reported differences in frequency of stressors.

\section{Did the study setting matter?}

We assumed that patients referred to psychiatry would have significantly higher rates of stressors than those referred to neurology. In fact, the difference was much less than expected (psychiatry OR $3 \cdot 7[1 \cdot 6-8 \cdot 495 \% \mathrm{CI}]$ and neurology OR $2 \cdot 9$ [1.6 to $5 \cdot 3]$ ).

Did the selection of control group matter?

The most important factor for the interpretation of results, of those we examined, was the choice of comparator group. There was a significant difference in results when the comparator group were healthy controls (OR 8.6 [4.9-15.0 95\% CI]) compared to any form of disease control. Surprisingly however, the choice 'disease comparator' had little impact and there was a similar strength of association irrespective of whether the comparator was neurological (OR 2.5 $[1 \cdot 5-4 \cdot 3$ 95\% CI]) or psychiatric controls (OR 2·0 [1·1-3.6 95\% CI]) (Figure 2).

\section{Did study quality matter?}

Notably, we did not find any differences between studies with a high quality rating and those with a low rating.

\section{Did the methodology for assessing stressors matter?}

We compared data from only those studies that used the well-validated Life Events and Difficulties Schedule $53,56,57$ (OR 4·3 [1·4-13·2 95\% CI]). This showed that the LEDS not surprisingly led to higher rates of reporting of stressors than other less rigorous methodologies such as questionnaires (OR 2·1 [0·5-8.7 95\% CI])(Figure 4)]).

\section{Did the association differ between children and adults?}

We found that the strength of the association in children for stressors (OR 13.4 [5•8-15.0 95\% $\mathrm{CI}]$ ) was much stronger than in adults (OR 2.9 [1.8-4.6 95\% CI]), although numbers in the paediatric studies were low (Figure 2).

Did the timing of stressor in relation to symptom onset affect the results? 
Studies that examined the occurrence of stressful life events that occurred immediately preceeding symptom onset found an increased risk when comparing cases to controls (OR 2.8 [1.4-6.0 95\% CI])(Figure 4), but when we looked at studies examining the occurrence of life events throughout adult life the results were equivocal (OR $2 \cdot 2[0 \cdot 9-5 \cdot 495 \% \mathrm{CI}]$ ). The broad confidence intervals may reflect the high frequency of stressful life events as a normal occurrence in adult life.

Figure 4

Analysis of the time period in which stressful life events occurred (without specification of the association with symptom onset) did not significantly affect the results, with studies assessing events of recent time periods ( 3 months or less before assessment) (OR 2.4 [1·3-4.4 95\% CI]) showing a similar strength of association to those occurring over a longer time period of 12 months before assessment (OR 3.3 [0.9-11.7 95\% CI]). However, we would caution that there was considerable heterogeneity (I2 90\% [75-94\%, 95\% CI])(Figure 4), and one of the highest quality studies that looked at multiple timepoints leading up to symptom onset found an increasing rate of severe events with increasing proximity to symptom onset ${ }^{56}$.

\section{Publication Bias}

We produced funnel plots that we visually inspected for all our analyses. Overall, there was no evidence of publication bias. Egger's bias statistics were non-significant for all summary statistics. The funnel plots are shown in Appendix B. 


\section{Discussion}

We found an increased rate of childhood and adult stressful life events and maltreatment in patients with FND compared to controls. The strength of the association was significantly higher when the comparison was with healthy controls (OR 8.6) compared to neurological (OR 2.5) or psychiatric (OR 2.0) control groups. A variable but, in some studies, substantial proportion of patients were found, who did not report an identifiable stressor.

Odds ratios can be difficult to interpret as they refer to the probability of two events being associated as opposed to the actual increased risk of that event. As an approximate guide for the reader the majority of odds ratios quoted (i.e. between 2-4) would be seen as a small to medium effect. The OR seen in relation to emotional neglect, or the effect of stressors on presentations of FND in children would be regarded as large effects ${ }^{64}$.

Emotional neglect had a stronger association with the development of FND, whether the neglect took place in childhood or adult life, than the more traditionally described physical or sexual abuse. Higher quality studies tended to find a slightly stronger association but the quality of study and setting did not have the impact we might have expected.

However, emotional neglect is believed to be less prevalent in the population in general than some of the other risk factors studied $20-26$. When looked at as population attribution fractions, which takes account of the population prevalence of the risk factor as well as the relative risk increase in the individual, we found that physical abuse in both childhood and adulthood may have the largest population attributable risk (16.9\% and 14.6\% respectively) whereas emotional neglect had an attributable risk of $15 \cdot 1 \%$ in childhood and $11 \%$ in adult life. Childhood sexual abuse accounted for an attributable risk of $8 \cdot 7 \%$ of adult cases and sexual abuse in adult life for $4 \cdot 8 \%$.

Our meta-analysis was strong in terms of identifying the appropriate papers but the metaanalytic methodology we used has some limitations. As described in the methods section we used a rationally derived hierarchy to choose one pair of data to avoid duplicate data appearing in the summary statistic. This has clear benefits in providing an objective and replicable way to deal with multiple data points. The sensitivity analysis should detect and account for any differences in the choice of data points but our choices were selective and may have influenced results. There were also limitations to the quality of the underlying literature. The quality of 
studies in this field was generally only fair with a median quality rating of 5/11 on our modified Newcastle Ottawa scale; although there were notable exceptions. Most individual studies were too small to find conclusive results and heterogenity was high. Despite that our meta-analyses and sensitivity analyses showed a reassuring picture of relatively consistent results given the diverse range of settings and methodologies, suggesting realiable conclusions.

Most studies used self-report questionnaires, which, although quicker and cheaper to use, are generally less sensitive. By contrast exhaustive enquiry into a wide range of possible stressors and gaining extensive details of the context of the subject's life, as done in a 'gold standard' interview method such as the LEDS63, did lead to higher rates of reporting of stressors. Whichever method was used, a proportion of FND patients reported no stressors.

The study of stressors has been largely one of retrospective assessment. For childhood stressors there are well documented problems with recall bias which can lead to either over- or underreporting65,66, but, for stressors occurring in adulthood, some retrospective methods have been validated with independent verification of remembered events ${ }^{67,68}$ - for example up to 5 years with the LEDS ${ }^{63}$. We have summarised the problems and challenges in studying stressors in FND in Box 1.

\section{Box 1. Methodological Issues with Studies of Stressful Life Events and Maltreatment in} FND

\section{Case finding and recruitment:}

- Diagnostic suspicion bias - some patients may have been given the diagnosis because they experienced stressors, when others without stressors may have just been left as 'blackouts ?cause'

- Misdiagnosis - published studies suggest this is rare

- Recruitment bias - those seen in psychiatric clinics may have experienced more stressors and may present with psychiatric comorbidity

- Patients with FND as defined strictly by DSM-IV or earlier would by definition have to have a "conflict" or "other stressors". In fact, few studies appeared to adhere to DSM.

- Making a diagnosis of FND may alter a patient's review of his/her life history and cause an erroneous reassessment of humdrum events as substantial stressors.

Sample sizes: small sample sizes unlikely to find a significant result even if the effect is there 
(type 2 error)

Blinding: only nine studies measure outcome blind to the diagnosis

Confounding with comorbidities: possibly the case for depression and anxiety. May also be true for personality disorder and other variables only partially dependent on FND

Interviewer factors: a patient may not trust the interviewer or feel ready to disclose events to them. It is argued that stressors will only be disclosed following prolonged clinical engagement and build up of trust. However, against this it should be noted our results found that those studies which assessed reported stressors solely on the basis of clinical contact had the lowest rates of detection and those which used a very comprehensive structured measure, like the LEDS, had the highest. What was less clear was the nature of the clinical contact and whether that included patients who had had prolonged psychotherapy.

\section{Multiple different measures of stressors}

\section{Difficulties of stressful life event studies in general}

- Recall bias can occur in both directions - patients may overly recall negative vs positive events, others may have experienced terrible maltreatment but deny it in interviews and questionnaires.

- Contextualizing events - stressful life events take on meaning because of the context in which they occur. Only contextualised methods such as the LEDS assess events in this way. Even when they do it is very hard to blind.

- Some studies were not specific regarding the time frame of stressors and appear to have included also those after symptom onset.

- Symptom specific events such as conflict over speaking out in dysphonia study may be prevalent but hidden in general questionnaires assessing stressors.

\section{Exposure not usually corroborated with external records}

Heterogeneity - if high then considerable caution is warranted when interpreting results of meta-analysis

Publication Bias - negative studies not published.

\section{Implications}

We do not claim our study is definitive on the topic of stressors and FND. There are limitations to the evidence. However, it should be noted that, for better or worse, this is the full extent of the case control evidence linking stressors to FND. It may come as a surprise to many clinicians what the extent, and the limitations, of the evidence is for such a well engrained theory. 
There are important clinical and research implications from our findings. For the clinician faced with an individual patient, stressful life events and maltreatment should still be considered as a potential aetiological factor in the development of FND and, when present, a potential treatment target. However, a proportion of patients do not report any such experiences, and such experiences are common in the population in general, therefore, whilst potentially aetiologically relevant, these exposures cannot be regarded as necessary in order to reach a diagnosis. Further, clinicians should not assume the patient is consciously or unconsciously failing to report stressors if none are forthcoming after thorough questioning. Like most clinicians practicing in the field we have had the experience of patients denying exposure to maltreatment only to disclose it down the line, but perhaps less memorable are the patients who go through treatment, often recovering and in whom no such history is ever disclosed. Our results suggest a proportion of patients report no such stressors, and our experience suggests excessive zeal in searching out maltreatment can be just as harmful as a complete lack of interest. Of note, emotional neglect is associated with a higher individual risk than physical or sexual abuse. Finally, it is clear from our results that more detailed assessment of stressors results in a higher disclosure rate; it is not a task that should be rushed. Given the lack of diagnostic weight attached to these variables this part of the assessment may often be better left to future appointments but this will vary depending on circumstances ${ }^{69}$.

This systematic review supports the decision to remove the need for a recent stressor from the diagnostic criteria for FND in DSM-5 and suggests that ICD-11 would benefit from following this approach. The diagnosis should be made on the basis of the history plus inconsistent and incongruent neurological signs. However, the review confirms the aetiological importance of stressors as risk factors. The implications are that neurologists and psychiatrists (and psychologist/psychotherapist) will continue to be essential for the field. In terms of ICD-11 it is therefore imperative that the condition is coded in both ' $F$ ' and ' $G$ ' codes- although we long for the day when we drop this dualistic approach and bring ICD into the $21^{\text {st }}$ century with a unitary code for clinical brain sciences disorders. Work on the relationship between stressful life events and maltreatment and illness behaviour, as well as altered brain functioning in FND will move our understanding forward regarding potential mechanisms ${ }^{70,71}$.

\section{Conclusion}

This review has aggregated data from 34 studies totaling 1405 patients and there is consistency in the findings that emerge that exposure to stressful life events and maltreatment in childhood 
and adult life is associated with an increase in the risk of FND, but not all FND patients have experienced identifiable stressors.

\section{Research in context}

\section{Evidence before this study}

In the last decade there has been a marked upsurge in interest in conversion (functional neurological) disorders. Largely dismissed during the latter part of the 20th century as an historical entity that was usually the product of misdiagnosis, there is now high quality evidence that such disorders are common, disabling and can be diagnosed accurately. The dominant view of aetiology had been that these symptoms arose as a consequence of the 'conversion' of psychic distress in to physical symptoms. With new research came new theories of aetiology; in particular increasingly sophisticated models of mechanism based in neurosciences. These theories challenged the dominant view of psychological stressors being 'converted' into physical symptoms. One view is that these new studies were complementary and simply explained the mechanism of conversion. Others took the stance that this was an alternate mechanism and that conversion disorder could occur in the absence of identifiable exposure to stressors. DSM-5 took the latter view and was explicit that the presence of such stressors was no longer required, although paradoxically went for a compromise name 'conversion (functional neurological symptoms) disorder'. This did not meet with universal approval and passionate debate exists, but has often been shaped by individuals citing case examples from their own practice and less attention has been paid to the existing data from case control studies. As a group of clinical researchers who have been involved in this debate but from opposing perspectives we sought to systematically review the available literature. We searched PubMed and Science Direct for case control studies from 1965 to end 2016, with the search terms ("psychogenic" OR "conversion disorder" OR "non-epileptic") AND ("abuse" OR "life event") AND ("control" OR "controlled" OR "case-control'). This search was supplemented by reviewing the reference lists of eligible studies and previous reviews. After removing duplicates and ineligible studies, we included 34 studies in our systematic review and meta-analysis.

\section{Added value of this study}

This review provides the most comprehensive aggregation of the evidence from case-control studies since 1965. We covered the full phenotype of conversion (functional neurological) 
disorder including both seizure disorders and motor/ sensory disorders. We examined the rate of stressful life events and of different types of maltreatment in childhood and adult life in conversion (functional neurological) patients and in both healthy and disease controls. In addition to a qualitative review of individual studies, we quantitatively evaluated the association between stressors and FND, and importantly conducted subgroup and sensitivity analyses to investigate sources of bias in order to understand the limitations to our data.

\section{Implications of all the available evidence}

Our results show that there is an increased rate of childhood and adult stressful life events and maltreatment, particularly emotional neglect, in patients with conversion (functional neurological) disorder compared to controls. The association was stronger in cases of childhood onset and when one compared to healthy as opposed to disease controls. However, a proportion of cases report no stressors. We concluded that stressors are aetiologically relevant to the development of conversion (functional neurological) disorder and therefore a potential treatment target, but exposure to such stressors is not an essential diagnostic feature. Our findings support the changes to DSM-5 and have implications for ICD-11.

Contributors

JS, AC, TN, SA, AD, RK, KR had the idea for the study. LL, JS, AC contributed to the study conception and design. LL and JS did the systematic literature research and selected studies for inclusion, TN, JP and KR contributed additional literature researches. LL extracted data, JP checked all data for accuracy. LL and JP assessed the quality of the studies, AC resolved any disagreements. LL and JS did statistical analyses, JS made the figures. LL wrote the first draft of the manuscript and AC, JS did redrafts. ST critically reviewed the analyses and the manuscript. All authors critically revised the manuscript for important intellectual content. All authors contributed and approved the final draft.

\section{Declaration of interests}

Lea Ludwig has nothing to disclose, Joëlle A. Pasman has nothing to disclose, Tim Nicholson has nothing to disclose, Selma Aybek has nothing to disclose, Anthony S. David has nothing to 
disclose, Sharon Tuck has nothing to disclose, Richard Kanaan has nothing to disclose, Karin Roelofs has nothing to disclose, Alan Carson: paid editor of JNNP, paid independent testimony in court on a range of related topics; Jon Stone: created and maintains

http://www.neurosymptoms.org self-help site for patients. Paid independent testimony in court including relating to functional disorders.

Acknowledgments

ASD and TN are supported by the NIHR Biomedical Research Centre at the South London and Maudsley NHS Foundation Trust and the Institute of Psychiatry, Psychology and Neuroscience, King's College London. JS is supported by a NHS Scotland Career Research Fellowship.

References

1. Stone, J. et al. Who is referred to neurology clinics? - The diagnoses made in 3781 new patients. Clin. Neurol. Neurosurg. 112, 747-751 (2010).

2. Stone, J., Warlow, C. \& Sharpe, M. The symptom of functional weakness: a controlled study of 107 patients. Brain 133, 1537-51 (2010).

3. American Psychiatric Association. (2000). Diagnostic and statistical manual of mental disorders (4th ed., text rev.). Washington, DC: Author.

4. Briquet, P. \& Baillière, J.-B. Traité clinique et thérapeutique de l'hystérie. VII, 724 (1859).

5. Breuer, J. \& Freud, S. Studies on Hysteria. (Hogarth Press, 1895).

6. Roelofs, K. \& Spinhoven, P. Trauma and medically unexplained symptoms towards an integration of cognitive and neuro-biological accounts. Clin. Psychol. Rev. 27, 798-820 (2007).

7. Sharpe, D. \& Faye, C. Non-epileptic seizures and child sexual abuse: a critical review of the literature. Clin. Psychol. Rev. 26, 1020-1040 (2006).

8. Fiszman, A., Alves-Leon, S. V., Nunes, R. G., D’Andrea, I. \& Figueira, I. Traumatic events and posttraumatic stress disorder in patients with psychogenic nonepileptic seizures: a critical review. Epilepsy Behav. 5, 818-25 (2004).

9. Paras, M. L. et al. Sexual abuse and lifetime diagnosis of somatic disorders: a systematic review and meta-analysis. JAMA 302, 550-61 (2009).

10. Afari, N. et al. Psychological trauma and functional somatic syndromes: a systematic review and meta-analysis. Psychosom. Med. 76, 2-11 (2014). 
11. Pareés, I. et al. Physical precipitating factors in functional movement disorders. J. Neurol. Sci. 338, 174-7 (2014).

12. Stone, J. et al. The role of physical injury in motor and sensory conversion symptoms: a systematic and narrative review. J. Psychosom. Res. 66, 383-390 (2009).

13. Nicholson, T. R. J. Studies In Conversion Disorder - Testing the psychological model \& Freudian theories. (King's College London, 2012).

14. Roelofs, K. \& Pasman, J. in Handbook of Clinical Neurology (eds. Hallett, M., Stone, J. \& Carson, A.) 139, 139-155 (2016).

15. Wells, G. A. et al. The Newcastle-Ottawa Scale (NOS) for assessing the quality of nonrandomised studies in meta-analyses. (2011). Available at:

http://www.medicine.mcgill.ca/rtamblyn/Readings/The Newcastle - Scale for assessing the quality of nonrandomised studies in meta-analyses.pdf. (Accessed: 13th September 2015)

16. Borenstein, M., Hedges, L. V., Higgins, J. P. T., \& Rothstein, H. R. (2009). Converting Among Effect Sizes. In Introduction to Meta-Analysis (pp. 45-47). Chichester, UK.

17. StatsDirect Ltd. StatsDirect statistical software. Available at: http://www.statsdirect.com England: StatsDirect Ltd. 2013.

18. Higgins, J. P. T., Thompson, S. G., Deeks, J. J. \& Altman, D. G. Measuring inconsistency in meta-analyses. BMJ 327, 557-60 (2003).

19. Calculation of population attribution fractions. Available at: http://sphweb.bumc.bu.edu/otlt/MPHModules/EP/EP713_Association/EP713_Association_print.html. (Accessed: 28th February 2017)

20. Stoltenborgh, M., van IJzendoorn, M. H., Euser, E. M. \& Bakermans-Kranenburg, M. J. A Global Perspective on Child Sexual Abuse: Meta-Analysis of Prevalence Around the World. Child Maltreat. 16, 79-101 (2011).

21. Abrahams, N. et al. Worldwide prevalence of non-partner sexual violence: a systematic review. Lancet (London, England) 383, 1648-54 (2014).

22. Sørbø, M. F., Grimstad, H., Bjørngaard, J. H., Schei, B. \& Lukasse, M. Prevalence of sexual, physical and emotional abuse in the Norwegian mother and child cohort study. BMC Public Health 13, 186 (2013).

23. Stoltenborgh, M., Bakermans-Kranenburg, M. J., van IJzendoorn, M. H. \& Alink, L. R. A. Cultural-geographical differences in the occurrence of child physical abuse? A meta-analysis of global prevalence. Int. J. Psychol. 48, 81-94 (2013).

24. Stoltenborgh, M., Bakermans-Kranenburg, M. J. \& van Ijzendoorn, M. H. The neglect of child neglect: a meta-analytic review of the prevalence of neglect. Soc. Psychiatry Psychiatr.

Epidemiol. 48, 345-55 (2013).

25. Gilbert, R. et al. Burden and consequences of child maltreatment in high-income countries. Lancet 373, 68-81 (2009).

26. Desmarais, S. L., Reeves, K. A., Nicholls, T. L., Telford, R. P. \& Fiebert, M. S. Prevalence of Physical Violence in Intimate Relationships, Part 1: Rates of Male and Female Victimization. 
Partner Abuse 3, 140-169 (2012).

27. Akyuz, G., Kugu, N., Akyuz, A. \& Dogan, O. Dissociation and childhood abuse history in epileptic and pseudoseizure patients. Epileptic Disord. 6, 187-92 (2004).

28. Almis, B. H., Cumurcu, B. E., Unal, S., Ozcan, a C. \& Aytas, O. The neuropsychological and neurophysiological profile of women with pseudoseizure. Compr. Psychiatry 54, 649-57 (2013).

29. Alper, K., Devinsky, O., Perrine, K., Vazquez, B. \& Luciano, D. Nonepileptic seizures and childhood sexual and physical abuse. Neurology 43, 1950-3 (1993).

30. Arnold, L. M. \& Privitera, M. D. Psychopathology and trauma in epileptic and psychogenic seizure patients. Psychosomatics 37, 438-43 (1996).

31. Bakvis, P. et al. Trauma, stress, and preconscious threat processing in patients with psychogenic nonepileptic seizures. Epilepsia 50, 1001-11 (2009).

32. Berkhoff, M., Briellmann, R. S., Radanov, B. P., Donati, F. \& Hess, C. W. Developmental background and outcome in patients with nonepileptic versus epileptic seizures: a controlled study. Epilepsia 39, 463-9 (1998).

33. Betts, T. \& Boden, S. Diagnosis, management and prognosis of a group of 128 patients with non-epileptic attack disorder. Part I. Seizure 1, 19-26 (1992).

34. Binzer, M., Stone, J. \& Sharpe, M. Recent onset pseudoseziures - clues to aetiology. Seizure 13, 146-155 (2004).

35. Dikel, T. N., Fennell, E. B. \& Gilmore, R. L. Posttraumatic stress disorder, dissociation, and sexual abuse history in epileptic and nonepileptic seizure patients. Epilepsy Behav. 4, 644-650 (2003).

36. Jawad, S. S. M. et al. Psychiatric morbidity and psychodynamics of patients with convulsive pseudoseizures. Seizure 4, 201-206 (1995).

37. Kaplan, M. J. et al. Comparisons of childhood trauma, alexithymia, and defensive styles in patients with psychogenic non-epileptic seizures vs. epilepsy: Implications for the etiology of conversion disorder. J. Psychosom. Res. 75, 142-6 (2013).

38. Kuyk, J., Spinhoven, P., van Emde Boas, W. \& van Dyck, R. Dissociation in temporal lobe epilepsy and pseudo-epileptic seizure patients. J. Nerv. Ment. Dis. 187, 713-20 (1999).

39. Litwin R \& Cardena E. Demographic and Seizure variables, But not Hypnotizability or Dissociation, Differentiated Psychogenic from Organic Seizures. J. Trauma Dissociation 1, 99-122 (2000).

40. McDade, G. \& Brown, S. W. Non-epileptic seizures: management and predictive factors of outcome. Seizure. 1, 7-10 (1992).

41. Mökleby, K. et al. Psychiatric Comorbidity and Hostility in Patients with Psychogenic Nonepileptic Seizures Compared with Somatoform Disorders and Healthy Controls. Epilepsia 43, 193-198 (2002).

42. Ozcetin, A. et al. Childhood trauma and dissociation in women with pseudoseizure-type conversion disorder. Nord. J. Psychiatry 63, 462-8 (2009).

43. Plioplys, S. et al. A multisite controlled study of risk factors in pediatric psychogenic 
nonepileptic seizures. Epilepsia 55, 1739-47 (2014).

44. Proença, I. C. G. F., Castro, L. H. M., Jorge, C. L. \& Marchetti, R. L. Emotional trauma and abuse in patients with psychogenic nonepileptic seizures. Epilepsy Behav. 20, 331-333 (2011).

45. Reilly, J., Baker, G. A., Rhodes, J. \& Salmon, P. The association of sexual and physical abuse with somatization: characteristics of patients presenting with irritable bowel syndrome and non-epileptic attack disorder. Psychol. Med. 29, 399-406 (1999).

46. Salmon, P., Al-Marzooqi, S. M., Baker, G. \& Reilly, J. Childhood Family Dysfunction and Associated Abuse in Patients With Nonepileptic Seizures. Psychosom. Med. 65, 695-700 (2003).

47. Say, G. N., Tasdemir, H. A., Akbas, S., Yüce, M. \& Karabekiroglu, K. Self-esteem and psychiatric features of Turkish adolescents with psychogenic non-epileptic seizures: a comparative study with epilepsy and healthy control groups. Int. J. Psychiatry Med. 47, 41-53 (2014).

48. Scévola, L. et al. Psychiatric disorders in patients with psychogenic nonepileptic seizures and drug-resistant epilepsy: a study of an Argentine population. Epilepsy Behav. 29, 155-60 (2013).

49. Testa, S. M., Krauss, G. L., Lesser, R. P. \& Brandt, J. Stressful life event appraisal and coping in patients with psychogenic seizures and those with epilepsy. Seizure 21, 282-7 (2012).

50. Tojek, T. M., Lumley, M., Barkley, G., Mahr, G. \& Thomas, A. Stress and other psychosocial characteristics of patients with psychogenic nonepileptic seizures. Psychosomatics 41, 221-6 (2000).

51. Barnett, C. Conversion reactions and psychophysiologic disorders: a comparative study. Psychiatry Med. 2, 205-2020 (1971).

52. Chabrol, H., Peresson, G. \& Clanet, M. Lack of specificity of the traditional criteria for conversion disorders. Eur. Psychiatry 10, 317-9 (1995).

53. Kozlowska, K., Scher, S. \& Williams, L. M. Patterns of emotional-cognitive functioning in pediatric conversion patients: implications for the conceptualization of conversion disorders. Psychosom. Med. 73, 775-88 (2011).

54. Roelofs, K., Keijsers, G. P., Hoogduin, K. A., Naring, G. W. \& Moene, F. C. Childhood abuse in patients with conversion disorder. Am. J. Psychiatry 159, 1908-1913 (2002).

55. Steffen, A., Fiess, J., Schmidt, R. \& Rockstroh, B. 'That pulled the rug out from under my feet!' - adverse experiences and altered emotion processing in patients with functional neurological symptoms compared to healthy comparison subjects. BMC Psychiatry 15, 133 (2015).

56. Nicholson, T. R. et al. Life events and escape in conversion disorder. Psychol. Med. 46, 2617 26 (2016).

57. Kranick, S. et al. Psychopathology and psychogenic movement disorders. Mov. Disord. 26, 1844-50 (2011).

58. Binzer, M. \& Eisemann, M. Childhood experiences and personality traits in patients with motor conversion symptoms. Acta Psychiatr. Scand. 98, 288-295 (1998).

59. Baker, J., Ben-Tovim, D., Butcher, A., Esterman, A. \& McLaughlin, K. Psychosocial risk 
factors which may differentiate between women with Functional Voice Disorder, Organic Voice Disorder and a Control group. Int. J. Speech. Lang. Pathol. 15, 1-17 (2012).

60. House, A. O. \& Andrews, H. B. Life events and difficulties preceding the onset of functional dysphonia. J.Psychosom.Res. 32, 311-319 (1988).

61. Roelofs, K., Spinhoven, P., Sandijck, P., Moene, F. C. \& Hoogduin, K. a L. The impact of early trauma and recent life-events on symptom severity in patients with conversion disorder. J. Nerv. Ment. Dis. 193, 508-514 (2005).

62. World Health Organization - Metrics: Population attribution fractions. Available at: http://www.who.int/healthinfo/global_burden_disease/metrics_paf/en/. (Accessed: 4th July 2017)

63. Brown, G. W. \& Harris, T. O. Social Origins of Depression. A Study of Psychiatric Disorder in Women. (Routledge, 1978).

64. Chen, H., Cohen, P. \& Chen, S. How Big is a Big Odds Ratio? Interpreting the Magnitudes of Odds Ratios in Epidemiological Studies. Commun. Stat. - Simul. Comput. 39, 860-864 (2010).

65. Hardt, J. \& Rutter, M. Validity of adult retrospective reports of adverse childhood experiences: Review of the evidence. J. Child Psychol. Psychiatry Allied Discip. 45, 260-273 (2004).

66. Reuben, A., Moffitt, T.E., Caspi, A., Belsky, D.W., Harrington, H.L., Schroeder, F., Hagan, S., Ramrakha, S., Poulton, R., Danese, A. Lest we forget: Comparing retrospective and prospective assessments of adverse childhood experiences in the prediction of adult health. $J$. Child Psychol. Psychiatry (2016). doi:in press

67. Tennant, C., Smith, A., Bebbington, P. \& Hurry, J. The contextual threat of life events: the concept and its reliability. Psychol. Med. 9, 525 (1979).

68. Neilson E, Brown GW, M. M. Myocardial Infarction, in Life events and illness. Guildford Press (1989).

69. Carson, A., Lehn, A., Ludwig, L. \& Stone, J. Explaining functional disorders in the neurology clinic: a photo story. Pract. Neurol. 16, 56-61 (2016).

70. Aybek, S. et al. Neural correlates of recall of life events in conversion disorder. JAMA psychiatry 71, 52-60 (2014).

71. Maurer, C. W. et al. Impaired self-agency in functional movement disorders. Neurology 87, 10.1212/WNL.0000000000002940 (2016). 